\title{
Accelerated Sarcopenia as an Initial Manifestation of Cushing's Disease
}

\author{
L.Pichaipillai ${ }^{1}$, M.Kumar ${ }^{1}$, D.Tiong ${ }^{1}$, S.Pathmanathan ${ }^{1}$, C.Higham ${ }^{1}$ \\ Department of Endocrinology, Christie NHS Foundation Trust, Manchester, UK ${ }^{1}$
}

Introduction: Cushing's Syndrome usually presents with a phenotype including central obesity, striae, hypertension and diabetes. We describe here a case with an atypical presentation of Cushing's Syndrome.

Case: A 73 year old lady was referred to the neurologists with a year's history of progressive limb wasting, weakness and recurrent falls. Following investigations including a normal CK, EMG (suggested myopathic process), nerve conduction studies (no evidence of large fibre neuropathy) and muscle biopsy (atrophic change compatible with on-going neurogenic process), she was diagnosed with accelerated sarcopenia. She had a 4 year history of hypertension and Type 2 diabetes and morning cortisol was $750 \mathrm{nmol} / \mathrm{L}$. At this stage she was referred to the endocrinology team. Clinical features included progressive weight loss, immobility, severe muscle wasting and friable skin with extensive bruising and breakdown.

Fig 1
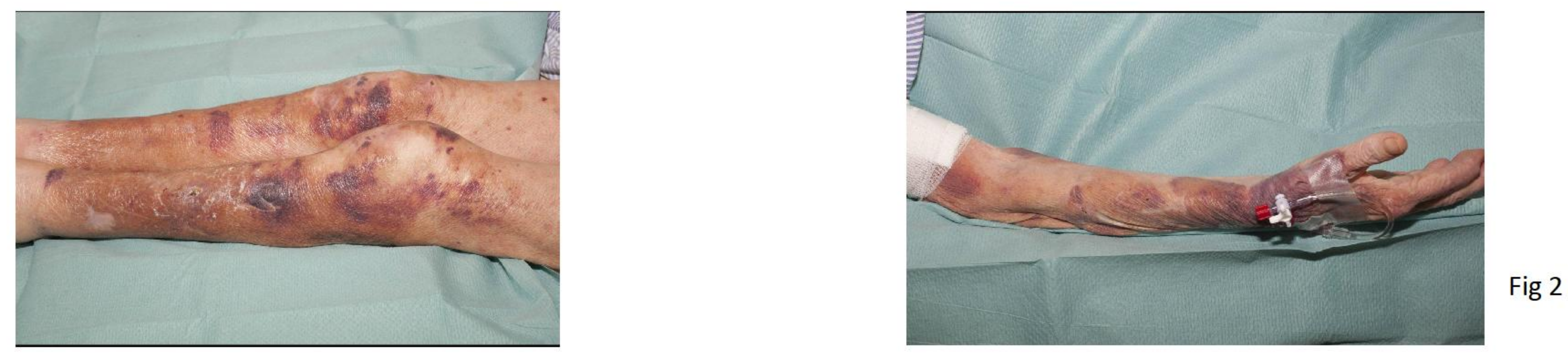

Results: Endocrine investigations (18 months post initial presentation) revealed:

- UFC $1227 \mathrm{nmols} / 24 \mathrm{hrs}(0-165)$

- Midnight salivary cortisol $45.1 \mathrm{nmols} / \mathrm{l}(0-2.6)$

- Non-suppressed cortisol following LDDST at $493 \mathrm{nmol} / \mathrm{L}$,

- ACTH 18ng/L (0-46)

- Normal serum potassium

- Initial MRI pituitary was normal.

- CT whole body showed no evidence of malignancy.

- Dynamic pituitary MRI revealed area of reduced signal within the pituitary-consistent with a microadenoma.

Fig 3
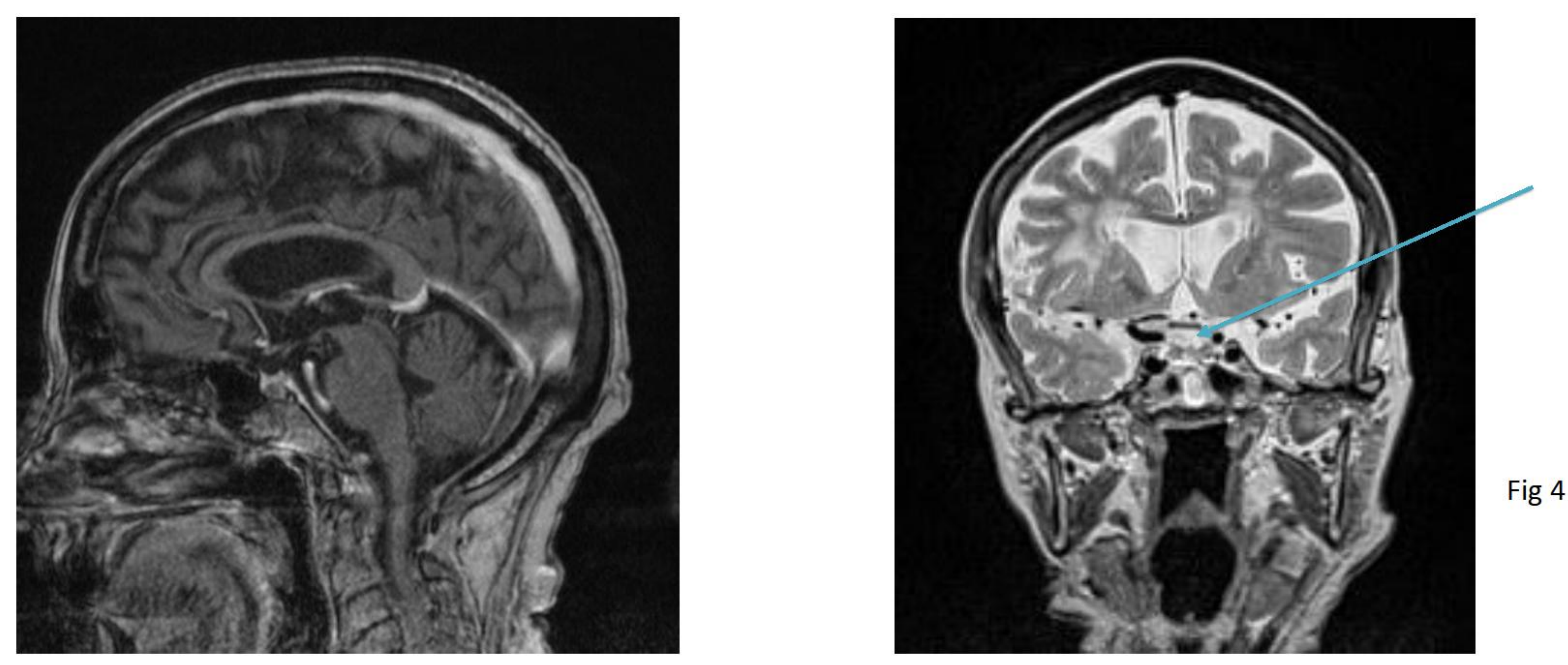

A diagnosis of Cushing's disease was made and the patient was commenced on Metyrapone. On 1gm tds her mean cortisol on day curve done by mass spectrometry was $390 \mathrm{nmol} / \mathrm{L}$. Antihypertensives and metformin were discontinued due to clinical improvement. Concurrent intensive physiotherapy lead to the regaining of limited mobility.

Discussion: Cushing's disease can rarely present with severe atypical clinical features that can mimic pathology of various systems, on this occasion accelerated sarcopenia, warranting neurological investigations. Sarcopenia refers to low muscle mass and loss of muscle function. Glucocorticoids inhibit protein synthesis and stimulate protein degradation in skeletal muscles. IGF1 is decreased and myostatin is increased and these changes result in protein catabolism, muscle atrophy, and, ultimately, weakness. It is important to consider and recognise this condition early in order to prevent extreme phenotypes, where regaining full recovery may be prolonged or unattained. 